\title{
Current Trends in Streptococcus pneumoniae Infections and Their Treatment
}

\author{
Avinash K. Shetty $\cdot$ Yvonne A. Maldonado
}

Published online: 19 June 2013

(C) Springer Science + Business Media New York 2013

\begin{abstract}
Invasive pneumococcal disease (IPD) is a major cause of pediatric morbidity and mortality in resource-rich and low and middle-income countries (LMICs). Streptococcus pneumoniae can cause a wide spectrum of invasive and non-invasive disease. In resourcerich countries, implementation of 7-valent pneumococcal conjugate vaccine (PCV7) has been a significant public health achievement. Dramatic decrease in pediatric IPD incidence occurred after PCV7 implementation, but emergence of multi-drug-resistant non-vaccine serotypes (especially 19A) remains a concern. The recently introduced 13-valent PCV (PCV13) has the potential to further reduce the burden of IPD due to serotypes not contained in PCV7. Given the diversity of pneumococcal serotypes and the possibility of serotype replacement disease, innovative approaches to development of pneumococcal vaccines are needed. Continued surveillance of pneumococcal serotypes and antimicrobial resistance is crucial to evaluate the impact of PCV13 vaccine. Improved access to PCVs is crucial to reduce morbidity and mortality related to IPD in LMIC.
\end{abstract}

Keywords Streptococcus pneumoniae - Invasive disease · Treatment

\footnotetext{
A. K. Shetty $(\bowtie)$

Department of Pediatrics, Wake Forest School of Medicine, Medical Center Blvd, Meads Hall, 3rd Floor, Winston-Salem, NC 27157, USA

e-mail: ashetty@wakehealth.edu

Y. A. Maldonado

Department of Pediatrics, Stanford University School of Medicine, 300 Pasteur Drive, Stanford, CA, USA
}

\section{Introduction}

Invasive disease caused by Streptococcus pneumoniae (pneumococcus) is a major cause of morbidity and mortality in children $[1,2,3 \bullet, 4 \bullet \bullet]$. The World Health Organization (WHO) estimates that 1.2 million infants die of invasive pneumococcal disease (IPD) each year, primarily in low and middle-income countries (LMICs) [5, 6]. In 2000, S. pneumoniae accounted for 14.5 million episodes of serious disease in children aged $<5$ years resulting in 826,000 deaths representing $11 \%(8-12 \%)$ of all deaths in children aged 1-59 months (excluding deaths in HIV-infected children) [1]. Children aged $<2$ years, adults $\geq 65$ years of age, and immunocompromised individuals remain at highest risk for IPD, defined as isolation of S. pneumoniae from a normally sterile body fluid [7]. Seven pneumococcal serotypes $(1,5,6 \mathrm{~A}, 6 \mathrm{~B}, 14,19 \mathrm{~F}$, and $23 \mathrm{~F})$ cause most cases of IPD in children worldwide [2, 8, 9]. The extent of disease due to different serotypes can vary by age, ethnicity, country, time, and clinical presentation [4०0]. The emergence of multi-drug resistant (MDR) $S$. pneumoniae is a major concern $[10,11]$. The introduction of 7 -valent pneumococcal conjugate vaccine (PCV7) in the US in 2000 has resulted in a significant decline in the incidence of IPD in all ages, especially in children aged $<2$ years [4••, 12]. Reduction in IPD among adults was noted, likely due to herd immunity [3•]. The success of PCV7 was accompanied by emergence of non-PCV7 serotypes especially 19A, warranting the development of 13-valent PCV (PCV13) [13-16, 17०•]. In this article, we review the changing epidemiology, pathogenesis, clinical manifestations, treatment and prevention of $S$. pneumoniae infections in children.

\section{Etiology}

Streptococcus pneumoniae is an encapsulated, lancetshaped, alpha-hemolytic, catalase-negative Gram-positive 
diplococcus that is part of the human nasopharyngeal (NP) flora [18-20]. A total of 94 immunologically distinct capsular serotypes of $S$. pneumoniae have been described including the recently identified serotypes $6 \mathrm{C}, 6 \mathrm{D}, 20 \mathrm{~A} /$ $20 \mathrm{~B}$, and $11 \mathrm{E}[2,21]$. In the US, historically most IPD were associated with serotypes $4,6 \mathrm{~B}, 9 \mathrm{~V}, 14,18 \mathrm{C}, 19 \mathrm{~F}$, and $23 \mathrm{~F}$ contained within PCV7 [22, 23]. Prior to PCV7 introduction, penicillin-resistance in pneumococci was most commonly associated with serotypes 6A, 6B, 9V, 14, 19A, 19F, and 23F [24]. After PCV7 use, serotype 19A is the most frequent etiology of IPD, but has emerged in unvaccinated population causing acute otitis media (AOM) $[25,26]$.

\section{Epidemiology}

NP Carriage and Transmission

Streptococcus pneumoniae colonizes the nasopharynx, usually with other co-pathogens, such as Haemophilus influenzae and Moraxella catarrhalis [27]. Pneumococcus is ubiquitous and primarily transmitted from person to person via contact with respiratory droplets [28, 29]. Young children remain the primary source of transmission to household contacts and adults [3•]. The microorganism is generally not considered contagious, although community outbreaks can rarely occur in certain populations [30]. The precise period of communicability is unclear but likely to be $<24 \mathrm{~h}$ after initiation of appropriate therapy [28].

Pneumococcal carriage in the nasopharynx begins during the first weeks of life and peaks during the first 2 years of life [31, 32]. Infants become colonized with pneumococcus around 6 months of age [18]. Epidemiologic studies suggest that NP pneumococcal carriage is a dynamic process acting as a reservoir for person to person transmission $[19,20]$. Highest carriage rates (40-60\%) are observed in young children, especially those attending day care centers [6]. Carriage rates decrease with increasing age ranging from $12 \%$ in older children to $6-10 \%$ in adolescents and $3-4 \%$ in adults [33]. Carriage rates in children can vary from $21 \%$ in resource-rich settings to $>90 \%$ in LMIC [28]. Pneumococcal carriage rates are also affected by race, socioeconomic status, seasonality, overcrowding, exposure to day care centers and passive smoke [6, 32, 34]. In institutional settings and during winter, point prevalence and longitudinal studies have reported maximal carriage rates varying from $21-59$ to $65 \%$, respectively $[6,34]$.

Most individuals are colonized with one pneumococcal serotype at a time, although co-colonization by multiple serotypes can occur in up to $30 \%$ in settings with high pneumococcal carriage rates $[32,35]$. Duration of carriage can vary from days to months and is highly dependent on the serotype [36]. Some serotypes (e.g., serotype 1, 4, 5, and $7 F$ ) colonize the nasopharynx rarely or to a lesser degree than others [37•,38]. The duration of the first colonizing serotype is often the longest; reacquisition of the same serotype is possible [18]. After colonization with one of the pneumococcal serotypes, a new strain may replace other competing serotypes and persists for weeks to months [18].

Serotype-specific acquired immunity to pneumococcal carriage has been noted, which likely explains the varied colonization rates between children and adults [39]. Recent acquisition of a new serotype is an important predictor of development of subsequent IPD compared to duration of carriage or specific clonal genotypes [40]. The survival strategy of $S$. pneumoniae in humans is dependent on selection of specific clones with either an invasive phenotype or a persistent colonization phenotype (non-invasive phenotype) [29]. Low immunogenicity serotypes $(6,14,19$ and 23) can persist in the nasopharynx for longer duration, more likely to be reacquired, serving as a reservoir for transmission, promoting pneumococcal survival and harbor antimicrobial resistance [3•, 19].

\section{Pathogenesis}

NP colonization with $S$. pneumoniae is a critical first step in the pathogenesis of IPD [6, 20]. The direct link between colonization and subsequent disease manifestation in the same individuals has been supported by some longitudinal studies [18, 41]. In one study, $15 \%$ of children who acquired a new strain developed otitis media within 1 month of acquisition of the new strain [18]. The mechanism of infection is by hematogenous route or contiguous spread, with migration of bacteria from the nasopharynx to adjacent structures such as middle ear, sinuses, and airways [4••]. The complex mechanisms involved in clearance or invasive disease resulting from pneumococcal carriage needs further elucidation [20, 29].

A wide array of pneumococcal virulence factors work collaboratively to cause invasive disease [42]. The most crucial virulence determinant of $S$. pneumoniae is the polysaccharide capsule that mediates invasion. The capsular antigen enables the microorganism to exhibit antigen variation, confers serotype specificity, prevents mucosal clearance, shows antiphagocytic and adherence properties, and sterically inhibits complement and immunoglobulinbinding to host receptors [43]. Pneumococcal virulence factors such as the capsule and pneumococcal surface molecules such as choline binding protein A and phosphorylcholine play a dual role in colonization and virulence [20]. Capsular switching from vaccine serotype to nonvaccine serotype can occur as a result of genetic recombination and lead to serotype replacement disease [4••]. Following PCV7, vaccine escape serotypes have emerged 
due to recombinant exchanges [44]. The emergence of many clones of pneumococcal serotype 19A is a result of capsular switching [15]. Other factors such as lack of efficacy of PCV7 against serotype 19A, antimicrobial resistance, and clonal expansion have led to the genetic diversity of 19A and emergence worldwide as the predominant serotype causing IPD [15, 25, 45].

Incidence of IPD

During the pre-PCV7 era, S. pneumoniae accounted for an estimated 16,250 cases of IPD each year in children aged $<5$ years in the US; PCV7 serotypes $(4,6 \mathrm{~B}, 9 \mathrm{~V}, 14$, $18 \mathrm{C}, 19 \mathrm{~F}$, and $23 \mathrm{~F}$ ) accounted for $80 \%$ of cases of IPD in children aged $<5$ years, and for $50 \%$ of cases in individuals $\geq 6$ years of age $[24,46]$. The epidemiology of IPD changed significantly after infant PCV7 vaccination program [12, 14, 47, 48]. A population- and laboratory-based active bacterial core $(A B C)$ surveillance system report a reduction in incidence of IPD among children aged $<5$ years from 99 cases per 100,000 population during 1998-1999 to 21 cases per 100,000 population in 2008 (79\% reduction) $[17 \bullet \bullet]$. The overall reduction in IPD rates resulted from dramatic declines $(99 \%)$ in IPD due to PCV7 vaccine serotypes in children aged $<5$ years and an overall decline by $95 \%$ among individuals of all age groups $[49,50]$. In one report, of the 753 cases of IPD reported in children fully immunized with PCV7, vaccine failures were observed in $4 \%$ cases; the remainder of cases was caused by non-vaccine serotypes [51].

Since 2002, the overall IPD rates have been estimated at $\sim 22$ to 25 annual cases per 100,000 children aged $<5$ years [46]. Further, PCV7 proved to be a costeffective intervention given the estimated $\$ 10,400$ cost per life-year saved after taking into consideration the indirect effects on IPD incidence [52]. Indirect benefits of infant PCV7 was noted resulting in an overall reduction of $45 \%$ among unvaccinated individuals of all age groups due to herd immunity [47, 48, 53]. Based on 2009 data from the Centers for Disease Control and Prevention (CDC) [17••], overall rates of IPD have declined 34,14 , and $37 \%$ in persons aged $18-49,50-64$, and $\geq 65$ years, respectively. IPD due to PCV7 vaccine serotypes were observed in children aged $<5$ years and an overall decline by $95 \%$ among individuals of all age groups [49, 50].

Serotype Replacement and Emergence of Antimicrobial Resistance

Pneumococcal serotypes can vary with time, patient age and geographic setting [21]. Following PCV7 implementation in resource-rich countries, non-PCV7 serotypes have increased among asymptomatic carriers ("serotype replacement")
[13]. Also, an increase in incidence of IPD due to non-vaccine types (especially serotype 19A) was noted in the US and several other countries in Europe and Asia-Pacific region $[12,15,25,45,54]$. The absolute rate increase in IPD due to serotype $19 \mathrm{~A}$ is estimated at $\sim 5$ cases per 100,000 population compared with decline in the rate of IPD due to PCV7 serotypes $[55,56]$. In the US, most serotype 19A disease results from proliferation of preexisting sequence type (ST) 199, but a new strain, designated ST 320, is prevalent in many countries $[15,57]$. The factors governing serotype replacement are complex given the natural variability in IPD and pneumococcal serotypes over time and the absence of increase in non-PCV7 serotype replacement IPD with PCV7 use in some studies [19, 53, 58].

Antibiotic Resistance Trends Among Replacement Serotypes

Prior to implementation of PCV7, five serotypes contained in PCV7 comprised $80 \%$ of penicillin-non-susceptible isolates [24]. Following the PCV7 program, studies reported a $57 \%$ decrease in the overall incidence of IPD (and $81 \%$ decrease in children aged $<2$ years) due to nonsusceptible pneumococcal serotypes contained in the PCV7 $[17 \bullet \bullet, 59]$. In addition, erythromycin-resistant and MDR pneumococcal strains also declined [17••].

However, a corresponding increase in disease caused by penicillin-non-susceptible, non-PCV7 pneumococcal serotypes was observed $[12,15,17 \bullet \bullet, 57,59,60]$. Most cases penicillin-resistant pneumococcal infections are caused by seven serotypes $(6 \mathrm{~A}, 6 \mathrm{~B}, 9 \mathrm{~V}, 14,19 \mathrm{~A}, 19 \mathrm{~F}$, and $23 \mathrm{~F})$ of which five are included in PCV7 $(6 \mathrm{~B}, 9 \mathrm{~V}, 14,19 \mathrm{~F}$, and 23F) whereas all of these important serotypes are included in PCV13 [4*0]. In the US MDR pneumococcal serotype $19 \mathrm{~A}$ is a common cause of IPD in the post PCV7 era [3*, $4 \cdot \bullet, 12,17 \bullet \bullet, 25,61]$.

\section{Risk Factors for IPD}

Before PCV7, children aged $<2$ years were at highest risk for disease given the high carriage rates in this age group, and other contributory factors such as poor natural immunity to the polysaccharide capsule and increased risk of exposure to viral respiratory infections [4••, 19]. Table 1 summarized the various host, pathogen, and environmental

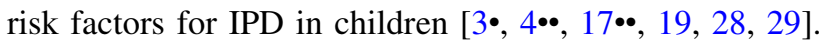

\section{Clinical Syndromes}

Streptococcus pneumoniae can cause a wide spectrum of clinical syndromes ranging from invasive disease (e.g., bacteremia, sepsis, meningitis, community-acquired 
Table 1 Risk factors for invasive pneumococcal disease

Host
Age $<2$ years pr $>65$ years
Ethnic groups/indigenous populations
African-American, Native American, Alaskan Eskimo
Micronesian
Aboriginal (Australia, New Zealand)
Absence of breastfeeding
No prior pneumococcal vaccinations
Co-morbid disease (e.g., cerebrospinal fluid leaks, cardiac or
renal disease, diabetes mellitus), cochlear implants
Antecedent viral respiratory infections (e.g., influenza)
Primary and secondary immune deficiency
human immunodeficiency virus infection
sickle cell disease
congenital or acquired asplenia
complement deficiencies
congenital or acquired antibody deficiency
malignancy or other forms of immunosuppression
Pathogen
Recent acquisition of new pneumococcal virulent strain
Environment
Childhood day care attendance
Wecent antibiotic use
Parental smoking
Overcrowding situations
Pintason

Data from $[3 \bullet, 4 \bullet \bullet, 17 \bullet \bullet, 19,28,19]$

pneumonia) to other common localized infections (e.g., otitis media, sinusitis). Table 2 depicts the common and uncommon clinical presentations of pneumococcal disease in children [4••, 22, 23, 61-63]. The incubation period may vary depending on the infection type, but can range from 1 to 3 days [28].

\section{Bacteremia}

Historically, pneumococcal bacteremia in infants and young children was a commonly reported disease [4••]. In the pre-PCV7 era, in children aged $\leq 4$ years, the annual incidence of pneumococcal bacteremia was $\sim 242$ cases per 100,000 individuals; infants aged $\leq 12$ months were at greatest risk ( $\sim 72 \%$ of reported cases) [64]. A variety of clinical presentations may be associated with bacteremia including meningitis, pneumonia, osteomyelitis or septic arthritis as well as otitis media [4••].

In the post-PCV7 era, the incidence of pneumococcal bacteremia has declined significantly in both children and adults [4••, 46, 48, 53]. However, studies have noted an increase in percentage of episodes of bacteremia caused by vaccine-related pneumococcal serotypes (those of the same
Table 2 Clinical spectrum of pneumococcal disease in children

\begin{tabular}{ll}
\hline Common presentations & Unusual presentations \\
\hline Otitis media and sinusitis & Infective endocarditis \\
Bacteremia & Periorbital cellulitis \\
Sepsis & Buccal cellulitis \\
Meningitis & Primary peritonitis \\
Pneumonia & Pericarditis \\
Osteomyelitis and septic arthritis & Mastoiditis \\
& Hemolytic-uremic syndrome \\
& Neonatal sepsis \\
\hline
\end{tabular}

Data from [4••, 22, 23, 61-63]

serogroup but not of the same serotype as PCV7) from $6 \%$ in the pre-PCV period to $35 \%$ in the post-PCV7 period [65]. In a recent $\mathrm{ABC}$ surveillance report, a reduction in the rate of bacteremia without focus source from $67.9 \%$ in the pre-PCV7 period to $10.1 \%$ in the post-PCV7 period was noted [46].

Pyogenic Meningitis

Globally, meningitis due to $S$. pneumoniae is estimated to cause $>60,000$ deaths in children aged $<5$ years [1]. $S$. pneumoniae is the most common cause of pyogenic meningitis in the US, with a bimodal age distribution [66]. During post-PCV7 era, rates of pneumococcal meningitis decreased significantly among children and adults [12, 14, 48, 67]. In 2004, data from the Nationwide Inpatient Sample from 1994 to 2004 indicated that S. pneumoniae was the most common pathogen causing bacterial meningitis accounting for 21,396 hospitalizations and 2,684 deaths (12.5\%) [67]. Following infant PCV7, there was a $66 \%$ decrease in hospitalization rates for pneumococcal meningitis per 100,000 individuals ( $7.7 \%$ in 1994-1997 to 2.6 in 2001-2004) in children under the age of 2 years. In children aged 2-4 years, there was a $51.5 \%$ decrease in hospitalizations rates per 100,000 individuals $(0.9 \%$ in 1994-1997 to 0.5 in 2001-2004). In addition, hospitalization rates due to pneumococcal meningitis also decreased by $33 \%$ for individuals $>65$ years of age [67]. Overall, PCV7 prevented an estimated 3,330 hospitalizations and 394 deaths related to pneumococcal meningitis in persons of all ages during 2001-2004 [67].

In a population-based surveillance project conducted from 1998 to 2005 at eight US sites, the overall rate of pneumococcal meningitis due to PCV7 serotypes decreased significantly (from $59 \%$ in 1998-1999 to $23 \%$ in 2004-2005) [12]. However, non-PCV7 serotype-associated disease rates increased significantly (from $28 \%$ in 1998-1999 to $65 \%$ in 2004-2005), with most increase attributed to serotypes 19A and 22F [12]. Despite the observation of serotype 
replacement disease, the overall incidence of pneumococcal meningitis due to PCV7 serotypes has declined by $30 \%$ to 0.79 cases per 100,000 people [12].

The spread of $S$. pneumoniae to the meninges typically occurs via the hematogenous route $[4 \bullet \bullet, 32]$. The clinical features can vary from an insidious onset of illness after an upper respiratory tract infection to a rapidly fulminant course resulting in death within $24 \mathrm{~h}$ of presentation [4*0]. Substantial morbidity and mortality can occur despite early recognition of illness. Neurologic sequelae (e.g., sensorineural hearing loss, paresis, and cognitive impairment) has been reported in $25-56 \%$ of survivors, and fatalities can occur in $5-15 \%$ of cases [4••].

\section{Pneumonia}

Streptococcus pneumoniae is the most common cause of CAP in children and adults worldwide [36]. In Latin America and Caribbean, where PCV7 was not implemented in national vaccination programs during 1980-2008, a recent meta-analysis found that serotype 14 was the most common cause of pneumococcal CAP, followed by serotypes 1 and 5 [68]. In the post-PCV7 era, pneumococcal serotype 1 is the most prevalent etiologic agent of pneumococcal CAP, followed by serotypes 19A, 3 and $14[69,70]$. Risk factors for pneumococcal pneumonia include primary or secondary immunodeficiency disorders, sickle cell disease, nephrotic syndrome, chronic lung disorders, chronic smoke inhalation, malnutrition, and viral respiratory infections, especially influenza [4••, 29]. Bacteremia may be present in $20-40 \%$ of all cases of pneumococcal pneumonia [4••, 50].

The clinical presentation of pneumococcal pneumonia can vary from a mild non-specific respiratory tract infection to a severe illness characterized by fever, cough, and dyspnea needing hospitalization [4••, 29]. Complications can occur in $40-50 \%$ of hospitalized children including pleural effusion, empyema, necrotizing pneumonia and lung abscess [22, 32]. Bacteremic pneumonia is more severe disease resulting in acute respiratory failure, empyema, and meningitis [4••]. In a recent meta-analysis, the risk of mortality was found to be higher in patients with bacteremic pneumonia caused by pneumococcal serotypes $3,6 \mathrm{~A}, 6 \mathrm{~B}, 9 \mathrm{~N}, 19 \mathrm{~A}, 19 \mathrm{~F}$, and $23 \mathrm{~F}$ than patients infected with serotype 14 whereas lower mortality was associated with serotypes $1,4,5,7 \mathrm{~F}$, and 8 than 14 [71].

Studies have shown a modest impact of PCV7 on the incidence of pneumococcal CAP [4••, 72]. During the postPCV era, a decrease in hospitalizations rates and outpatient visits for community-acquired pneumonia has been reported [72]. In one CDC report from pre-PCV7 period (1997-1999) to 2006 , hospitalization rate for all-cause pneumonia declined $35 \%$ among children under age 2 years but not in children aged 2-4 years [72]. A Cochrane Database Systematic
Review found a $27 \%$ pooled PCV7 vaccine efficacy for preventing radiograph-proven pneumonia and $6 \%$ for clinically diagnosed pneumonia in children aged $<2$ years [73].

Although PCV7 resulted in significant decreases in the rates of IPD and pneumococcal pneumonia in children, there has not been an effect on the incidence of empyema [4••]. Studies have documented an increase in the incidence of empyema resulting in hospitalizations $[74,75]$. In one report, a 2.17-fold overall increase in the rates of empyema complicating pneumococcal CAP was observed; in children $\leq 2$ years of age there was a 2.01 -fold increase (from 3.5 cases per 100,000 children in 1996-1998 to 7 cases per 100,000 children in 2005-2007) and in children 2-4 years of age, there was a 2.81-fold increase (from 3.7 cases per 100,000 children in $1996-1998$ to 10.3 cases per 100,000 children in 2005-2007) [74]. In another study, a $70 \%$ increase in the annual rate of empyema-associated hospitalization was noted from 1997 to 2006 (increase from 2.2 cases per 100,000 children in 1997 to 3.7 cases per 100,000 children in 2006) [75].

Non-PCV7 serotypes 1, 3, and 19A are most commonly associated with cases of pediatric pneumonia [25]. In a recent US study, the most common serotypes associated with empyema was serotype $3(26.5 \%)$ and 19A (22.4\%), followed by serotypes $7 \mathrm{~F}(14.3 \%)$ and $1(12.2 \%)$ [76]. Non-PCV7 serotype 1 has been linked to pediatric empyema cases [77, 78]. Genotypic analysis using multi-locus sequence typing of pneumococcal serotype 1 isolates have shown that ST 306, a newly detected genotype, emerged after PCV7 implementation whereas STs-227, -228, and -304 were previously existing genotypes [78, 79]. The potential of pneumococcal serotype 1 to cause empyema may be attributed to the presence of zwitterionic polysaccharides present in the pneumococcal capsule [37•].

\section{Bone and Joint Infections}

In children with microbiologically confirmed orthopedic infections, pneumococcus is the etiology in $\sim 4 \%$ of osteomyelitis and $\sim 20 \%$ of septic arthritis [4••]. Bone and joint infections constitute $3-6 \%$ of IPD $[4 \bullet \bullet, 80,81]$. The pathogenesis involves hematogenous spread of microorganisms, and the most commonly involved bones include the femur and humerus [80]. Rarely, other bones such as the vertebra may be infected [4*0]. The knee and hip are most commonly affected in septic arthritis [80]. Pneumococcal osteomyelitis and septic arthritis may be associated with positive blood culture for S. pneumoniae in approximately $50 \%$ of cases [80]. After PCV7 was introduced, there was a $50 \%$ decrease in pediatric osteomyelitis and septic arthritis due to $S$. pneumoniae compared with prePCV7 period [16]. 
Hemolytic-Uremic Syndrome

Other unusual presentations of IPD include HUS [55, 62]. The clinical course in pneumococcal HUS can be severe with higher dialysis requirements and mortality rate compared with typical HUS following enterotoxigenic Escherichia coli [32]. In the pre-PCV era, serotype 14 was most commonly implicated, but during the post-PCV7 era, serotype 3 has emerged as a major cause followed by serotypes $1,7 \mathrm{~F}$ and $19 \mathrm{~A}[62,82]$.

Otitis Media and Sinusitis

AOM is one of the most frequent infections encountered in pediatric practices, accounting for an estimated 13 million episodes each year among US children and resulting in health care expenditures of billions of dollars [83]. AOM often occurs in infants and young children aged between 3 and 24 months after a preceding viral upper respiratory infection, which results in impairment of the mucociliary apparatus and Eustachian tube dysfunction [84]. The peak age incidence for AOM is 3-24 months, which coincides with the peak incidence of community-acquired viral infections in children [85]. Otoscopic findings are crucial in making a clinical diagnosis of AOM [86].

Streptococcus pneumoniae is the most common microorganism causing AOM (35-40\% of cases) followed by non-typeable $H$. influenzae (30-35\%) and $M$. catarrhalis (15\%) [83]. After introduction of PCV7, there has been a decrease in ambulatory visits for otitis media, rates of frequent otitis media, and tympanostomy-tube placement among young children [87]. In addition, a transient decline in the prevalence of $S$. pneumoniae infection and a relative increase in $H$. influenzae infection was noted during the post-PCV7 period [83, 88]. However, the emergence of vaccine-related replacement serotype 19A with high-level amoxicillin resistance resulting in treatment failure has been a concern [89]. In the PCV7 era, considerable variability has been noted in the prevalence of penicillinresistant $S$. pneumoniae [83]. In addition, $\beta$-lactamase producing strains of 35-45\% of $\mathrm{H}$. influenzae strains account for 35-45\% of cases while almost $100 \%$ of $M$. catarrhalis isolates are $\beta$-lactamase positive [90].

\section{Treatment}

The emergence of MDR S. pneumoniae has complicated the treatment of pneumococcal infections [10, 11, 25, 26]. Over the past three decades, pneumococcal strains have emerged worldwide that are not susceptible to $\beta$-lactams (penicillin and ampicillin, third-generation cephalosporins such as ceftriaxone and cefotaxime), and other antimicrobial agents such as macrolides (including erythromycin, clarithromycin, and azithromycin), clindamycin, and trimethoprim-sulfamethoxazole (TMP-SMX) [10, 11]. In the PCV7 era, the emergence and spread of MDR CC320/271 strains with serotype 19A is a major therapeutic challenge [25, 91].

Antimicrobial Susceptibility Testing and Penicillin Breakpoints

The role of pharmacokinetic and -dynamic properties has proven invaluable to determine breakpoints for all antimicrobial agents used to treat pneumococcal infections [11]. In 2008, new breakpoints for penicillin $\mathrm{G}$ to treat non-meningeal infections were published by the Clinical and Laboratory Standards Institute [92] defining susceptibility and non-susceptibility for meningeal and non-meningeal pneumococcal isolates [93]. The breakpoints for intravenous penicillin $\mathrm{G}$ to treat meningitis were unchanged (susceptible, $\leq 0.06 \mu \mathrm{g} / \mathrm{ml}$; resistant, $\geq 0.12 \mu \mathrm{g} / \mathrm{ml}$ ). However, the breakpoints for intravenous penicillin $G$ to treat non-meningeal infection were changed (susceptible, $\leq 2.0 \mu \mathrm{g} / \mathrm{ml}$; intermediate, $\geq 4.0 \mu \mathrm{g} /$ $\mathrm{ml}$; resistant, $\geq 8.0 \mu \mathrm{g} / \mathrm{ml}$ ). The breakpoints for oral penicillin to treat non-meningeal pneumococcal infection remain the same (susceptible, $\leq 0.06 \mu \mathrm{g} / \mathrm{ml}$; intermediate, $0.12-1.0 \mu \mathrm{g} /$ $\mathrm{ml}$; resistant, $\geq 2.0 \mu \mathrm{g} / \mathrm{ml}$ ) [92, 93]. With the exception of meningitis, in vitro results are not predictive of treatment outcome in pneumococcal disease [11].

During 2006-2007, the CDC estimated that the percentage of IPD non-meningeal pneumococcal isolates defined as susceptible, intermediate, and resistant to penicillin changed from 74.7, 15.0, and $10.3 \%$ under the old breakpoints to 93.2, 5.6, and $1.2 \%$, respectively, under the revised 2008 breakpoints [93]. Using the revised penicillin susceptibility breakpoint of $\leq 2 \mu \mathrm{g} / \mathrm{ml}$ for non-meningeal infections, the SENTRY Antimicrobial Surveillance Program found that $93 \%$ of all pneumococcal isolates would be susceptible to parenteral penicillin [94]. However, using the revised penicillin susceptibility breakpoint of $\leq 0.06 \mu \mathrm{g} / \mathrm{ml}$ for meningeal infections, only $68 \%$ of isolates were susceptible [94]. The SENTRY Program in 2008 found that rates of resistance to erythromycin, clindamycin, and TMP-SMX were 37.1, 20.3, and $22.5 \%$, respectively among respiratory pneumococcal isolates [95].

Mechanisms of Antimicrobial Resistance

The mechanism of penicillin resistance in pneumococci is alteration in the cell wall penicillin-binding proteins (PBPs) $[10,96]$. Alterations in PBP1a, 2x and 2b results in increased MICs to $\beta$-lactam agents and decrease drug affinity [10]. With the exception of meningitis, higher concentrations of $\beta$-lactam antibiotics at non-meningeal 
sites of infection (e.g. otitis media, sinusitis, pneumonia) can bind and inhibit the enzyme and retain clinical effectiveness against penicillin-non-susceptible pneumococcal strains [10]. Resistance to quinolone and TMP-SMX to $S$. pneumoniae occurs from mutations within the bacterial genome [10].

\section{Treatment of Pneumococcal Meningitis}

Antimicrobial therapy in pneumococcal meningitis is challenging given the presence of pneumococcal strains non-susceptible to penicillin $G$, cefotaxime, ceftriaxone $[10,11,96]$. In addition, achievable therapeutic concentrations of antibiotics are lower in cerebrospinal fluid (CSF) [96]. Therefore, treatment of proven or suspected pneumococcal meningitis in infants aged $\geq 1$ month and children includes combination therapy with vancomycin plus cefotaxime or ceftriaxone [28, 96]. Combination therapy also provides optimal empiric coverage for other bacteria causing meningitis in children such as $N$. meningitidis. Patients who are allergic to penicillin or cephalosporin may be treated with vancomycin and rifampin [28]. Monotherapy with vancomycin or rifampin is not recommended because of concerns of achieving sustained bactericidal concentrations in the CSF (with vancomycin) and risk of development of resistance (with rifampin) [28]. Other alternative agents used for therapy of meningitis due to cephalosporin-resistant pneumococcal strains include carbapenems (e.g., imipenem, meropenem) [28].

Therapy can be adjusted once antibiotic susceptibility testing results are available. If the pneumococcal isolate is susceptible to penicillin (MIC $\leq 0.06 \mu \mathrm{g} / \mathrm{ml}$ ), vancomycin must be discontinued and penicillin, cefotaxime, or ceftriaxone should be continued alone [28]. For isolates nonsusceptible to penicillin (intermediate or resistant) but susceptible to extended-spectrum third-generation cephalosporins, vancomycin should be discontinued and cefotaxime or ceftriaxone must be continued [28]. For isolates non-susceptible to both penicillin and cefotaxime and ceftriaxone (intermediate or resistant) and susceptible to rifampin, addition of rifampin is recommended if there is clinical worsening after $24-48 \mathrm{~h}$ on vancomycin plus cefotaxime or ceftriaxone therapy, positive CSF culture for pneumococcus on repeat lumbar puncture or isolate showing high MIC ( $>4 \mu \mathrm{g} / \mathrm{ml})$ to cefotaxime or ceftriaxone [28]. Given the unreliable meningeal penetration of vancomycin, third-generation cephalosporin therapy is continued with vancomycin plus rifampin in cases of meningitis due to $S$. pneumoniae resistant to penicillin, cefotaxime and ceftriaxone [28]. In cases of meningitis caused by highly penicillin- or cephalosporin-resistant pneumococcal strains, use of fluoroquinolone (e.g., moxifloxacin) with in vitro activity against $S$. pneumoniae is an alternative therapeutic option for patients not responding to standard therapy; in such cases, fluoroquinolone must be used in combination with a third-generation cephalosporin or vancomycin [97]. The recommended dose of vancomycin therapy for bacterial meningitis is $60 \mathrm{mg} / \mathrm{kg} / \mathrm{day}$ in four divided doses, but to maintain a target trough concentration of about $15-20 \mu \mathrm{g} / \mathrm{ml}$ to achieve adequate CSF concentrations, the dose and frequency of vancomycin may have be individualized [98].

A repeat lumbar puncture to document sterility after $48 \mathrm{~h}$ of therapy is recommended in patients not responding to appropriate therapy, CSF culture positive for penicillin non-susceptible $S$. pneumoniae (by oxacillin disk or quantitative MIC testing) and pending results of cefotaxime or ceftriaxone quantitative susceptibility testing, and prior dexamethasone therapy, which may limit the ability to assess clinical response [28]. The recommended duration of parenteral antimicrobial therapy for uncomplicated meningitis is 10 days [28]. The role of adjunctive corticosteroid (dexamethasone) therapy in the treatment of pneumococcal meningitis in infants ( $>6$ weeks of age) and children is controversial [28]. If considered, dexamethasone should be administered before or concomitant with the first doses of antibiotics.

\section{Treatment of Non-meningeal IPD}

Previously healthy children with non-meningeal IPD (e.g., bacteremia, pneumonia) requiring hospitalizations (but not critically ill) can be treated with $\beta$-lactam antibiotics such as parenteral penicillin, ampicillin, cefuroxime, cefotaxime, and ceftriaxone [28]. Critically ill patients with suspected non-meningeal invasive disease due to pneumococcus (e.g., severe necrotizing or multilobar pneumonia with hypoxia or shock) must receive parenteral vancomycin plus a $\beta$-lactam agent (such as ceftriaxone or cefotaxime) to treat possible strains that are non-susceptible to penicillin, ceftriaxone or cefotaxime [28]. Vancomycin must be discontinued once the results of susceptibility testing become available and the organism is susceptible to penicillin or third generation cephalosporins. A pediatric infectious disease consultation is recommended for treatment of non-meningeal IPD due to pneumococcal strains that are non-susceptible to penicillin $\mathrm{G}$, cefotaxime or ceftriaxone.

\section{Acute Otitis Media}

For uncomplicated AOM and acute sinusitis, current American Academy of Pediatrics [99] and the American Academy of Family Physicians guidelines recommend high-dose amoxicillin (80-90 mg/kg/day divided twice daily) as the drug of choice in most instances. Given its excellent pharmacodynamic profile, high-dose amoxicillin 
can achieve adequate concentrations in the middle ear or sinus fluid that exceed the MICs to most penicillin-nonsusceptible pneumococcal strains and achieve bacteriologic eradication [84, 99]. In selected circumstances, patients with AOM may be observed without the need for antimicrobial therapy. The recommended duration of treatment for young infants with AOM or children with severe disease is 10 days, but in children older than 6 years of age who have mild or moderate disease, 5-7 days of therapy is sufficient [28, 99].

In the absence of clinical improvement, treatment failure must be reassessed at 3-5 days after initial therapy and prescribed alternative oral agents such as high-dose amoxicillin-clavulanate, cefdinir, cefpodoxime, or cefuroxime or a 3-day course of intramuscular (im) ceftriaxone [28]. These alternative agents have activity against penicillin-non-susceptible pneumococci as well as $\beta$-lactamase-producing $H$. influenzae and $M$. catarrhalis. To reduce the frequency of diarrhea, a 14:1 formulation of amoxicillin-clavulanate is recommended at $80-90 \mathrm{mg} / \mathrm{kg} / \mathrm{day}$ of the amoxicillin component. A 3-day course of im ceftriaxone can be used to treat patients who fail courses of oral antibiotic therapy [28].

Macrolide therapy with azithromycin or clarithromycin is reserved for patients with severe hypersensitivity reaction to $\beta$-lactams. In children with severe disease or those not responding to second-line therapy, obtaining a diagnostic culture via myringotomy or tympanocentesis is prudent for making appropriate therapeutic decisions [28, 84]. Pneumococcal serotype 19A has been implicated as an important otopathogen in cases of AOM treatment failure [89]. Clindamycin, rifampin, or other alternative antimicrobial agents are indicated for MDR resistant strains of $S$. pneumoniae [28].

\section{Alternative Antimicrobial Agents for Treatment of Resistant S. pneumoniae Infection}

In some cases, alternative agents (e.g., linezolid, quinupristin-dalfopristin or fluoroquinolones such as levofloxacin) may be indicated after careful consideration of the risks and benefits and in consultation with a pediatric infectious disease specialist. Linezolid is a synthetic oxazolidinone active in vitro against resistant Gram-positive bacteria, including MRSA, vancomycin-resistant enterococcus, and MDR $S$. pneumoniae [100]. Initially approved for clinical use by the FDA in 2000, linezolid is bacteriostatic and inhibits bacterial protein synthesis by binding to domain $\mathrm{V}$ of the $23 \mathrm{~S}$ ribosomal RNA of the 50S subunit [101]. Linezolid has been shown to be effective in the treatment of skin and skin-structure infections, bacteremia, or pneumonia in children but concerns about toxicity and potential development of resistance [101]. Linezolid is available in oral and parenteral formulations. Adverse effects include hematologic toxicity (anemia and thrombocytopenia) and peripheral neuropathy during chronic use [101].

\section{Newer Antimicrobial Agents}

The concern of evolving antimicrobial resistance to pneumococci and other Gram-positive pathogens has led to the development of several new antibiotics to treat CAP and other serious infections [102*0]. New antimicrobial agents available for treatment of resistant Gram-positive infections include daptomycin, tigecycline, and telavancin [100]. Ceftaroline is a novel fifth-generation cephalosporin with broad-spectrum of activity against both Gram-positive and -negative pathogens including MRSA and resistant pneumococci [100]. Newer agents in ketolides (a subclass of macrolides) under development include telithromycin, cethromycin, and solithromycin [102••]. However, most new agents currently under development are derivatives of currently existing classes of antimicrobial agents raising the concerns of cross-resistance [11].

\section{Prevention}

Although the PCV7 vaccination program stated in 2000 has made a substantial impact in preventing pneumococcal disease in the US, non-PCV7 serotype replacement disease has necessitated the development of PCV with expanded serotype coverage $[8,17 \bullet \bullet]$. In February 2010, a new PCV13 was recommended in place of PCV7 for routine pediatric immunization in the US from 6 weeks through 71 months of age [17••]. A single supplemental dose is recommended in all children aged 14-59 months who have been previously immunized with age-appropriate PCV7 series [17••]. The AAP Red Book [28] has published recommendations related to the use of PCV13 in children unimmunized or incompletely immunized with PCV13 as well as immunization of children 6-18 years of age with high-risk conditions.

In 2007, the ABC Surveillance Group reported that $64 \%$ of 427 IPD cases observed in studies were caused by pneumococcal serotypes contained in PCV7, especially serotype 19A (42\%) [17••]. PCV13 includes all the seven serotypes included in PCV7, with an additional six serotypes $(1,3,5,6 \mathrm{~A}, 7 \mathrm{~F}$, and $19 \mathrm{~A})$ that cause a majority of IPD [17••]. PCV13 has the potential to address the variation in serotype distribution, and further reduce the burden of IPD [8]. Data from the UK indicate that IPD due to serotypes contained in PCV13 has been reduced by $50 \%$ in children less than 2 years of age [16]. In LMIC, serotypes 1 and 5 are common causes of IPD. Due to efforts from the Global Alliance for Vaccines and Immunization, eight 
LMIC (Nicaragua, Honduras, Guyana, Sierra Leone, Kenya, and Democratic Republic of Congo, Mali, and Yemen) have introduced PCV13 during 2010-2011 [8]. A 10 -valent PCV has also been developed and gained WHO pre-qualification in $2009[4 \bullet \bullet, 8]$.

Given the diversity of pneumococcal serotypes, capsular switching potential, and the possibility of serotype replacement disease in both IPD and carriage, innovative approaches are needed to develop newer vaccines, such as conserved pneumococcal protein vaccines (CPPVs) [4••, 8, 19]. A number of CPPVs are currently being developed targeting combinations of conserved virulence proteins including pneumococcal surface protein A and pneumolysin, triple antigen vaccine based on antigens StKP, PcsB and PsaA, inactivated whole cell vaccine, and common protein vaccines $[4 \bullet \bullet, 8]$.

\section{Conclusion}

IPD remains an important cause of morbidity and mortality in children, especially in LMIC where PCV has not been implemented. NP carriage of $S$. pneumoniae is a precursor to disease. S. pneumoniae can cause a wide spectrum of invasive and non-invasive disease. In resource-rich countries, implementation of PCV7 has been a significant public health achievement. However, serotype replacement disease due to MDR non-vaccine serotypes remain a concern. The recently introduced PCV13 with expanded serotype coverage has the potential to further reduce the burden of IPD due to serotypes not contained in PCV7. Continued surveillance of pneumococcal serotypes and antimicrobial resistance is crucial to evaluate the impact of PCV13 vaccine. Improved access to PCVs is crucial to reduce morbidity and mortality related to IPD in LMIC.

Disclosure Avinash K. Shetty and Yvonne A. Maldonado declare no conflicts of interest.

Ethical Standards This article does not contain any studies with human or animal subjects performed by any of the authors.

\section{References}

Paper of particular interest, published recently, have been highlighted as:

- Of importance

•- Of major importance

1. O'Brien KL, Wolfson IJ, Watt JP, et al. Burden of disease caused by Streptococcus pneumoniae in children younger than 5 years: global estimates. Lancet. 2009;374:893-902.
2. Johnson HL, Deloria-Knoll M, Levine OS, et al. Systematic evaluation of serotypes causing invasive pneumococcal disease among children under five: the pneumococcal global serotype project. PLoS Med. 2010;7:e1000348.

3. - Lynch III JP, Zhanel GG. Streptococcus pneumoniae: epidemiology and risk factors, evolution of antimicrobial resistance, and impact of vaccines. Curr Opin Pulm Med. 2010;16:217-25. Comprehensive review of the epidemiology, risk factors and antimicrobial resistance of Streptococcus pneumoniae as well as impact of pneumococcal conjugate vaccines on disease burden.

4. - Tan TQ. Pediatric invasive pneumococcal disease in the United States in the era of pneumococcal conjugate vaccines. Clin Microbiol Rev. 2012;25:409-19. State of the art review of the epidemiology, clinical manifestations, and impact of the pneumococcal conjugate vaccines.

5. Berkley JA, Lowe BS, Isaiah MP, et al. Bacteremia among children admitted to a rural hospital in Kenya. N Engl J Med. 2005;352:39-42.

6. Bogaert D, De Groot R, Hermans R. Streptococcus pneumoniae colonization. Lancet Infect Dis. 2004;4:144-54.

7. Naheed A, Saha SK, Brieman RF, et al. Multihospital surveillance of pneumonia burden among children aged $<5$ years hospitalized for pneumonia in Bangladesh. Clin Infect Dis. 2009;48(Suppl 2):S82-9.

8. Jefferies JM, MacDonald E, Faust SN, Clarker SC. 13-Valent pneumococcal conjugate vaccine (PCV13). Hum Vaccines. 2011;7:1012-8.

9. Rodgers GL, Klugman KP. The future of pneumococcal disease prevention. Vaccine. 2011;29S:C43-8.

10. Jones RN, Jacobs MR, Sader HS. Evolving trends in Streptococcus pneumoniae resistance: implications for therapy of community-acquired pneumonia. Int $\mathrm{J}$ Antimicrob Agents. 2010;36:197-204.

11. Jacobs MR. Antimicrobial-resistant S. pneumoniae: trends and management. Expert Rev Anti-infect Ther. 2008;6:619-35.

12. Hsu HE, Shutt KA, Moore MR, et al. Effect of pneumococcal conjugate vaccine on pneumococcal meningitis. N Engl J Med. 2009;360:244-56.

13. Weinberger DM, Malley R, Lipsitch M. Serotype replacement in disease after pneumococcal vaccination. Lancet. 2011;378: 1962-73.

14. Kaplan SL, Mason EO Jr, Wald ER, et al. Decrease of invasive pneumococcal infections in children among 8 children's hospitals in the United States after the introduction of the 7-valent pneumococcal conjugate vaccine. Pediatrics. 2004;113:443-9.

15. Moore MR, Gertz RE Jr, Woodbury RL, et al. Population snapshot of emergent Streptococcus pneumoniae serotype 19A in the United States, 2005. J Infect Dis. 2008;197:1016-27.

16. Miller E, Andrews NJ, Waight PA, et al. Effectiveness of the new serotypes in the 13-valent pneumococcal conjugate vaccine. Vaccine. 2011;29:9127-31.

17. $\bullet$ Centers for Disease Control and Prevention. Prevention of pneumococcal disease among infants and children-use of the 13-valent pneumococcal conjugate vaccine and 23-valent pneumococcal polysaccharide vaccine. MMWR Recomm Rep. 2010;59(RR-11):1-18. Recommendations of the Advisory Committee on Immunization Practices us Diseases (ACIP) for the prevention of pneumococcal disease among infants and children less than 18 years of age.

18. Gray BM, Converse GM III, Dillon HC Jr. Epidemiologic studies of Streptococcus pneumoniae in infants: acquisition, carriage, and infection during the first 24 months of life. J Infect Dis. 1980;142:923-33.

19. Mehr S, Wood N. Streptococcus pneumoniae-a review of carriage, infection, serotype replacement and vaccination. Paediatr Respir Rev. 2012;13:258-64. 
20. Simell B, Auranen K, Kayhty H, et al. The fundamental link between pneumococcal carriage and disease. Expert Rev Vaccines. 2012;11:841-55.

21. Calix JJ, Dagan R, Pelton SI, Porat N, Nahm MH. Differential occurence of Streptococcus pneumoniae serotype 11E between asymptomatic carriage and invasive pneumococcal disease isolates reflects an unique model of pathogen microevolution. Clin Infect Dis. 2012;54:794-9.

22. Tan TQ, Mason EO Jr, Wald ER, et al. Clinical characteristics of children with complicated pneumonia caused by Streptococcus pneumoniae. Pediatrics. 2002;110:1-6.

23. Kaplan SL, Mason EO Jr, Barson WJ, et al. Outcome of invasive infections outside the central nervous system caused by Streptococcus pneumoniae isolates nonsusceptible to ceftriaxone in children treated with beta-lactam antibiotics. Pediatr Infect Dis J. 2001;20:392-6.

24. Centers for Disease Control and Prevention. Preventing pneumococcal disease among infants and young children: recommendations of the Advisory Committee on Immunization Practices (ACIP). MMWR. 2000;49(RR-9):1-38.

25. Kaplan SL, Barson WJ, Lin PL, et al. Serotype 19A is the most common serotype causing invasive pneumococcal infections in children. Pediatrics. 2010;125:429-36.

26. Dagan R, Givon-Lavi N, Leibovitz E, Greenberg D, Porat N. Introduction and proliferation of multidrug-resistant Streptococcus pneumoniae serotype 19A clones that cause acute otitis media in an unvaccinated population. J Infect Dis. 2009;199:776-85.

27. Sulikowska A, Grzesiowski P, Sadowy E, Fiett J, Hryniewicz W. Characteristics of Streptococcus pneumoniae, Haemophilus influenzae, and Moraxella catarrhalis isolated from the nasopharynges of asymptomatic children and molecular analysis of $S$. pneumoniae and $H$. influenzae strain replacement in the nasopharynx. J Clin Microbiol. 2004;42:3942-9.

28. American Academy of Pediatrics. Pneumococcal infections. In: Pickering LK, Baker CJ, Kimberlin DW, Long SS, editors. Red Book: 2012 Report of the Committee on Infectious Diseases. Elk Grove Village: American Academy of Pediatrics; 2012. p. $571-82$.

29. van der Poll T, Opal SM. Pathogenesis, treatment, and prevention of pneumococcal pneumonia. Lancet. 2009;374:1543-6.

30. Romney MG, Hull MW, Gustafson R, et al. Large community outbreak of Streptococcus pneumoniae serotype 5 invasive infection in an impoverished, urban population. Clin Infect Dis. 2008;47:768-74.

31. Hill PC, Cheung YB, Akisanya A, et al. Nasopharyngeal carriage of Streptococcus pneumoniae in Gambian infants: a longitudinal study. Clin Infect Dis. 2008;46:807-14.

32. Andrade AL, Toscano CM, Minamisava R, Costa PS. Pneumococcal disease manifestation in children before and after vaccination: What's new? Vaccine. 2011;29S:C2-14.

33. Cardozo DM, Nascimento-Carvalho CM, Andrade AL, et al. Prevalence and risk factors for nasopharyngeal carriage of Streptococcus pneumoniae among adolescents. J Med Microbiol. 2008;57:185-9.

34. Hill PC, Townend J, Antonio M, et al. Transmission of Streptococcus pneumoniae in rural Gambian villages: a longitudinal study. Clin Infect Dis. 2010;50:1468-76.

35. Turner $\mathrm{P}$, Hinds $\mathrm{J}$, Turner $\mathrm{C}$, et al. Improved detection of nasopharyngeal colonization by multiple pneumococcal serotypes by use of latex agglutination or molecular serotyping by microarray. J Clin Microbiol. 2011;49:1784-9.

36. Smith T, Lehmann D, Montgomery J, et al. Acquisition and invasiveness of different serotypes of Streptococcus pneumoniae in young children. Epidemiol Infect. 1993;111:27-39.

37. - Ritchie ND, Mitchell TJ, Evans TJ. What is different about serotype 1 pneumococci? Future Microbiol. 2012;7:33-46. This article reviews the unique characteristics of pneumococcal serotype 1 in relation to nasopharyngeal carriage and disease compared with other serotypes.

38. Sleeman KI, Griffiths D, Shackley F, et al. Capsular serotypespecific attack rates and duration of carriage of Streptococcus pneumoniae in a population of children. J Infect Dis. 2006;194: 682-8.

39. Weinberger DM, Dagan R, Givon-Lavi N, et al. Epidemiologic evidence for serotype-specific acquired immunity to pneumococcal carriage. J Infect Dis. 2008;197:1511-8.

40. Brueggemann AB, Griffiths DT, Meats E, et al. Clonal relationships between invasive and carriage Streptococcus pneumoniae and serotype-and clone-specific differences in invasive disease potential. J Infect Dis. 2003;187:1424-32.

41. Syrjanen RK, Auranen KJ, Leino TM, et al. Pneumococcal acute otitis media in relation to pneumococcal nasopharyngeal carriage. Pediatr Infect Dis J. 2005;24:801-6.

42. Mitchell AM, Mitchell TJ. Streptococcus pneumoniae: virulence factors and variation. Clin Microbiol Infect. 2010;16:411-8.

43. Nelson A, Roche AM, Gould JM, et al. Capsule enhances pneumococcal colonization by limiting mucus-mediated clearance. Infect Immunol. 2007;75:83-90.

44. Bruggemann AB, Pai R, Crook DQ, Beall B. Vaccine escape recombinants emerge after pneumococcal vaccination in the United States. PLoS Pathog. 2007;3:e168.

45. Reinert R, Jacobs MR, Kaplan SL. Pneumococcal disease caused by serotype 19A: review of the literature and implications for future vaccine development. Vaccine. 2010;28:4249-59.

46. Pilishvili T, Lexau C, Farley MM, et al. Active Bacterial Core Surveillance/Emerging Infections Program Network. Sustained reductions in invasive pneumococcal disease in the era of conjugate vaccine. J Infect Dis. 2010;201:32-41.

47. Grijalva CG, Griffin MR. Population-based impact of routine infant immunization with pneumococcal conjugate vaccine in the USA. Expert Rev Vaccines. 2008;7:83-95.

48. Whitney CG, Farley MM, Hadler J, et al. Decline in invasive pneumococcal disease after the introduction of protein-polysaccharide conjugate vaccine. N Engl J Med. 2003;348:1737-46.

49. American Academy of Pediatrics. Policy statement-recommendations for the prevention of Streptococcus pneumoniae infections in infants and children: use of 13-valent pneumococcal conjugate vaccine (PCV13) and pneumococcal polysaccharide vaccine (PPSV23). Pediatrics. 2010;126:186-90.

50. Whitney CG, Pilishvili T, Farley MM, et al. Effectiveness of seven-valent pneumococcal conjugate vaccine against invasive pneumococcal disease: a matched case-control study. Lancet. 2006;368:1495-502.

51. Park SY, Van Beneden CA, Pilishvili T, Martin M, Facklam RR, Whitley CG. Active Bacterial Core Surveillance Team. Invasive pneumococcal infections among vaccinated children in the United States. J Pediatr. 2010;156:478.e2-483.e2.

52. Ray GT, Pelton SI, Klugman KP, Strutton DR, Moore MR. Cost-effectiveness of pneumococcal conjugate vaccine: an update after 7 years of use in the United States. Vaccine. 2009;27:6483-94.

53. Poehling KA, Talbot TR, Griffin MR, et al. Invasive pneumococcal disease among infants before and after introduction of pneumococcal conjugate vaccine. JAMA. 2006;295:1668-74.

54. Techasaensiri C, Messina AF, Katz K, Ahmad N, Huang R, McCracken GH Jr. Epidemiology and evolution of invasive pneumococcal disease caused by multidrug resistant serotypes of 19A in the 8 years after implementation of pneumococcal conjugate vaccine immunization in Dallas, Texas. Pediatr Infect Dis J. 2010;29:294-300.

55. Byington CL, Samore MH, Stoddard GJ, et al. Temporal trends of invasive disease due to Streptococcus pneumoniae among 
children in the intermountain West: emergence of nonvaccine serogroups. Clin Infect Dis. 2005;41:21-9.

56. Hsu KK, Shea KM, Stevenson AE, Pelton SI. Changing serotypes causing childhood invasive pneumococcal disease: Massachusetts, 2001-2007. Pediatr Infect Dis J. 2010;29:251-6.

57. Ardanuy C, Rolo D, Fenoll A, Tarrago D, Calatayud L, Linares J. Emergence of a multidrug-resistant clone (ST320) among invasive serotype 19A pneumococci in Spain. J Antimicrob Chemother. 2009;64:507-10.

58. Harboe ZB, Benfield TL, Valentine-Branth P, et al. Temporal trends in invasive pneumococcus disease and pneumococcal serotypes over 7 decades. Clin Infect Dis. 2010;50:329-37.

59. Kyaw MH, Lynfield R, Schaffner W, et al. Effect of introduction of the pneumococcal conjugate vaccine on drug-resistant Streptococcus pneumoniae. N Engl J Med. 2006;354:1455-63.

60. Dagan R, Klugman KP. Impact of conjugate pneumococcal vaccines on antibiotic resistance. Lancet Infect Dis. 2008;8:785-95.

61. Ongkasuwan J, Valdez TA, Hulten KG, Mason EO Jr, Kaplan SL. Pneumococcal mastoiditis in children and the emergence of multidrug resistant serotype 19A isolates. Pediatrics. 2008;122: 34-9.

62. Copelovitch L, Kaplan BS. Streptococcus pneumoniae-associated hemolytic uremia syndrome: classification and the emergence of serotype 19A. Pediatrics. 2010;125:e174-82.

63. Kaplan SL, Mason EO Jr, Barson WJ, et al. Three-year multicenter surveillance of systemic pneumococcal infections in children. Pediatrics. 1998;102:538-45.

64. Jacobs NM, Lerdkachornsuk S, Metzger WI. Pneumococcal bacteremia in infants and children: a ten-year experience at the Cook County Hospital with special reference to the pneumococcal serotypes isolated. Pediatrics. 1979;64:296-300.

65. Steenhoff AP, Shah SS, Ratner AJ. Emergence of vaccinerelated pneumococcal serotypes as a cause of bacteremia. Clin Infect Dis. 2006;42:907-14.

66. Song JY, Nahm MH, Moseley MA. Clinical implications of pneumococcal serotypes: invasive disease potential, clinical presentations, and antibiotic resistance. J Korean Med Sci. 2013;28:4-15.

67. Tsai CJ, Griffin MR, Nuorti JP, Grijalva CG. Changing epidemiology of pneumococcal meningitis after the introduction of pneumococcal conjugate vaccine in the United States. Clin Infect Dis. 2008;46:1664-72.

68. Gentle A, Bardach A, Ciapponi A, et al. Epidemiology of community-acquired pneumonia in children of Latin America and the Caribbean: a systematic review and meta-analysis. Int $\mathrm{J}$ Infect Dis. 2012;16:e5-15.

69. Cilloniz C, Ewig S, Polverino E, et al. Pulmonary complications of pneumococcal community-acquired pneumonia: incidence, predictors, and outcomes. Clin Microbiol Infect. 2012;18:1134-42.

70. Bender JM, Ampofo K, Korgenski K, et al. Pneumococcal necrotizing pneumonia in Utah: Does serotype matter? Clin Infect Dis. 2008;46:1346-52.

71. Weinberger DM, et al. Association of serotype with risk of death due to pneumococcal pneumonia: a meta-analysis. Clin Infect Dis. 2010;51:692-9.

72. Centers for Disease Control and Prevention. Pneumonia hospitalizations among young children before and after introduction of pneumococcal conjugate vaccine-United States, 1997-2006. MMWR. 2009;58:1-4.

73. Lucero MG, et al. Pneumococcal conjugate vaccines for preventing vaccine-type invasive pneumococcal disease and X-ray defined pneumonia in children less than two years of age. Cochrane Database Syst Rev. 2009;CD004977.

74. Grijalva CG, Nuorti P, Zhu Y, Griffin MR. Increasing incidence of empyema complicating childhood community-acquired pneumonia in the United States. Clin Infect Dis. 2010;50: 805-13.

75. Li ST, Tancredi DJ. Empyema hospitalizations increased in US children despite pneumococcal conjugate vaccine. Pediatrics. 2010;125:26-33.

76. Yu J, Salamon D, Marcon N, Nahm MH. Pneumococcal serotypes causing pneumonia with pleural effusion in pediatric patients. J Clin Microbiol. 2011;49:534-8.

77. Resti M, Morionbdo M, Cortmiglia M, et al. Communityacquired bacteremia pneumococcal pneumonia in children: diagnosis and serotyping by real-time polymerase chain reaction using blood samples. Clin Infect Dis. 2010;51:1042-9.

78. Byington CL, Hulten KG, Ampofo K, et al. Molecular epidemiology of pediatric pneumococcal empyema from 2001 to 2007 in Utah. J Clin Microbiol. 2010;48:520-5.

79. Obando I, Munoz-Almagro C, Arroyo LA, et al. Pediatric parapneumonic empyema, Spain. Emerg Infect Dis. 2008;14: 1390-7.

80. Bradley JS, et al. Pediatric pneumococcal bone and joint infections. Pediatrics. 1998;102:1376-82.

81. Ross JJ, Saltzman CL, Carling P, Shapiro DS. Pneumococcal septic arthritis: review of 190 cases. Clin Infect Dis. 2003;36: 319-27.

82. Bender JM, Ampofo K, Byington CL, et al. Epidemiology of Streptococcus pneumoniae-induced hemolytic uremic syndrome in Utah children. Pediatr Infect Dis J. 2010;29:712-6.

83. Wald ER. Acute otitis media and acute bacterial sinusitis. Clin Infect Dis. 2011;52(Suppl 4):S277-83.

84. Pichichero ME. Otitis media. Pediatr Clin N Am. 2013;60: 391-407.

85. Chonmaitree T, Revai K, Grady JJ, et al. Viral upper respiratory tract infection and otitis media complication in young children. Clin Infect Dis. 2008;46:815-23.

86. Coker TR, Chan LS, Newberry SJ, et al. Diagnosis, microbial epidemiology, and antibiotic treatment of acute otitis media in children. JAMA. 2010;304:2161-9.

87. Poehling KA, Szilagyi PG, Grijalva CG, et al. Reduction of frequent otitis media and pressure-equalizing tube insertions in children after introduction of pneumococcal conjugate vaccine. Pediatrics. 2007;119:707-15.

88. Benninger MS. Acute bacterial rhinosinusitis and otitis media: changes in pathogenicity following widespread use of pneumococcal conjugate vaccine. Otolaryngol Head Neck Surg. 2008;138:274-8.

89. Critchley IA, Jacobs MR, Brown SD, et al. Prevalence of serotype 19A Streptococcus pneumoniae among isolates from U.S. children in 2005-2006 and activity of faropenem. Antimicrob Agents Chemother. 2008;52:2639-43.

90. Leibovitz E, Jacobs MR, Dagan R. Haemophilus influenzae: a significant pathogen in acute otitis media. Pediatr Infect Dis J. 2004;23:1142-52.

91. Beall BW, Gertz RE, Hulkower RL, et al. Shifting genetic structure of invasive serotype 19A pneumococci in the United States. J Infect Dis. 2011;203:1360-8.

92. Clinical and Laboratory Standards Institute. Performance standards for antimicrobial susceptibility testing: 18th informational supplement. CLSI Publication No. M100-S21. Wayne: Clinical and Laboratory Standards Institute; 2011.

93. Centers for Disease Control and Prevention. Effects of new penicillin susceptibility breakpoints for Streptococcus pneumoniae-United States, 2006-2007. MMWR. 2008;57:1353-5.

94. Weinstein MP, Klugman KP, Jones RN. Rationale for revised penicillin susceptibility breakpoints versus Streptococcus pneumoniae: coping with antimicrobial susceptibility in an era of resistance. Clin Infect Dis. 2009;48:1596-600. 
95. Jones RN, Sader HS, Moet GJ, Farrell DJ. Declining antimicrobial susceptibility of Streptococcus pneumoniae in the United States: report from the SENTRY Antimicrobial Surveillance Program (1998-2009). Diagn Microbiol Infect Dis. 2010;68:334-6.

96. American Academy of Pediatrics, Committee on Infectious Diseases. Therapy for children with invasive pneumococcal infections. Pediatrics. 1997;99:289-98.

97. Hameed N, Tunkel AR. Treatment of drug-resistant pneumococcal meningitis. Curr Infect Dis Rep. 2010;12:274-81.

98. Tunkel AR, Hartman BJ, Kaplan SL, et al. Practice guidelines for the management of bacterial meningitis. Clin Infect Dis. 2004;39:1267-84.

99. American Academy of Pediatrics, Clinical Practice Guideline. Subcommittee on management of acute otitis media. Pediatrics. 2004;113:1451-65.
100. Rivera AM, Boucher HW. Current concepts in antimicrobial therapy against select Gram-positive organisms: methicillinresistant Staphylococcus aureus, penicillin-resistant pneumococci, and vancomycin-resistant enterococci. Mayo Clin Proc. 2011;86:1230-42.

101. Chiappini E, Conti C, Galli L, de Martino M. Clinical efficacy and tolerability of linezolid in pediatric patients: a systematic review. Clin Ther. 2010;32:66-88.

102. •• Viasus D, Garcia-Vidal C, Carratala J. Advances in antibiotic therapy for community-acquired pneumonia. Curr Opin Pulm Med. 2013;19:209-15. This article reviews the advances in antibiotic therapy for community-acquired pneumonia focusing on development of new antimicrobial agents. 